\title{
Erratum to: Climate change under aggressive mitigation: the ENSEMBLES multi-model experiment
}

\author{
T. C. Johns $\cdot$ J.-F. Royer $\cdot$ I. Höschel $\cdot$ H. Huebener $\cdot$ E. Roeckner $\cdot$ E. Manzini $\cdot$ W. May $\cdot$ \\ J.-L. Dufresne $\cdot$ O. H. Otterå - D. P. van Vuuren · D. Salas y Melia $\cdot$ M. A. Giorgetta \\ S. Denvil $\cdot$ S. Yang $\cdot$ P. G. Fogli $\cdot$ J. Körper $\cdot$ J. F. Tjiputra $\cdot$ E. Stehfest $\cdot$ C. D. Hewitt
}

Published online: 25 June 2011

(c) Springer-Verlag 2011

\section{Erratum to: Clim Dyn (2011) \\ DOI: $10.1007 / \mathrm{s00382-011-1005-5}$}

Unfortunately, in the aforementioned contribution, Table 2 of this paper erroneously reported two separate rows of data for the BCM-C model for both the A1B and E1 scenarios, only one of which was correct in each case. The lower of the two rows of data for each scenario (i.e. those

The online version of the original article can be found under doi:10.1007/s00382-011-1005-5.

T. C. Johns $(\bowtie) \cdot$ C. D. Hewitt

Hadley Centre, Met Office, FitzRoy Road, Exeter EX1 3PB, UK

e-mail: tim.johns@metoffice.gov.uk

URL: http://www.metoffice.gov.uk

\section{J.-F. Royer - D. Salas y Melia}

Centre National de Recherches Météorologiques-Groupe d'Etude de l'Atmosphère Météorologique (CNRM-GAME Meteo-France CNRS), 42 Avenue G. Coriolis, 31057 Toulouse, France

I. Höschel · J. Körper

Institute for Meteorology, Freie Universität Berlin,

Carl-Heinrich-Becker-Weg 6-10, 12165 Berlin, Germany

H. Huebener

Hessian Agency for the Environment and Geology,

Rheingaustraße 186, 65203 Wiesbaden, Germany

E. Roeckner · E. Manzini - M. A. Giorgetta

Max Planck Institute for Meteorology, Bundesstrasse 53,

20146 Hamburg, Germany

E. Manzini

Istituto Nazionale di Geofisica e Vulcanologia, Bologna, Italy

E. Manzini · P. G. Fogli

Centro Euro-Mediterraneo per i Cambiamenti Climatici

(CMCC), Bologna, Italy corresponding to $\mathrm{T}$ changes of $2.44 \mathrm{~K}$ for $\mathrm{A} 1 \mathrm{~B}$ and $1.18 \mathrm{~K}$ for E1) contained correct data. The upper rows of data reported (i.e. those corresponding to $\mathrm{T}$ changes of $2.65 \mathrm{~K}$ for A1B and 1.38 for E1) contained some errors and should not have appeared. A corrected version of Table 2 appears below.

W. May $\cdot$ S. Yang

Danish Climate Centre, Danish Meteorological Institute, Lyngbyvej 100, 2100 Copenhagen, Denmark

\section{J.-L. Dufresne}

UMR 8539 CNRS, ENS, UPMC, Ecole Polytechnique,

Laboratoire de Météorologie Dynamique (LMD/IPSL),

75252 Paris Cedex 05, France

O. H. Otterå

Nansen Environmental and Remote Sensing Center,

Thormøhlensgt. 47, 5006 Bergen, Norway

O. H. Otterå

Uni. Bjerknes Centre, Allegt. 55, 5007 Bergen, Norway

O. H. Otterå · J. F. Tjiputra

Bjerknes Centre for Climate Research,

Allegt. 55, 5007 Bergen, Norway

D. P. van Vuuren

Utrech University, Utrech, The Netherlands

D. P. van Vuuren · E. Stehfest

Planbureau voor de Leefomgeving (PBL), Bilthoven,

The Netherlands 
Table 2 Changes in global annual mean temperature (T), precipitation (PR), cloud radiative forcing (SWCRF-shortwave, LWCRFlongwave, and $\mathrm{CRF}$ - net), atmospheric net radiative flux divergence
(AA) and net surface energy flux (FS) for the decade 2090-2099 in the A1B and E1 scenarios relative to 1990-1999

\begin{tabular}{|c|c|c|c|c|c|c|c|c|}
\hline & Model & $\mathrm{T}(\mathrm{K})$ & PR (mm/day) & SWCRF & LWCRF & $\mathrm{CRF}\left(\mathrm{W} / \mathrm{m}^{2}\right)$ & AA & FS \\
\hline \multirow[t]{10}{*}{ A1B } & ECHAM5-C & 3.28 & 0.152 & 0.99 & -0.72 & 0.27 & -3.16 & 1.92 \\
\hline & IPSL-CM4 & 3.15 & 0.171 & 3.03 & -1.54 & 1.48 & -4.63 & 1.08 \\
\hline & HadCM3C & 3.12 & 0.087 & & & & -2.79 & 0.82 \\
\hline & IPSL-CM4-LOOP & 3.06 & 0.187 & 2.89 & -1.36 & 1.53 & -4.81 & 1.22 \\
\hline & HadGEM2-AO & 2.97 & 0.052 & 0.82 & -0.71 & 0.11 & -0.30 & 0.94 \\
\hline & INGVCE & 2.80 & 0.146 & -0.85 & -0.62 & -1.47 & -1.86 & 1.26 \\
\hline & BCM2 & 2.48 & 0.136 & 1.10 & -0.85 & 0.25 & -2.92 & 1.06 \\
\hline & BCM-C & 2.44 & 0.134 & 1.03 & -1.00 & 0.04 & -2.73 & 0.60 \\
\hline & CNRM-CM3.3 & 1.95 & 0.105 & 1.26 & -1.50 & -0.24 & -1.81 & 0.78 \\
\hline & EGMAM+ & 1.76 & 0.045 & -0.93 & 0.48 & -0.45 & -0.30 & 0.86 \\
\hline \multirow[t]{10}{*}{ E1 } & INGVCE & 1.51 & 0.108 & 0.03 & 0.05 & 0.08 & -1.98 & 0.41 \\
\hline & IPSL-CM4 & 1.50 & 0.098 & 1.00 & -0.33 & 0.67 & -2.71 & 0.06 \\
\hline & HadCM3C & 1.41 & 0.080 & & & & -2.40 & -0.07 \\
\hline & HadGEM2-AO & 1.40 & 0.106 & 0.59 & 0.19 & 0.78 & -2.98 & 0.30 \\
\hline & ECHAM5-C & 1.40 & 0.083 & 0.83 & 0.00 & 0.83 & -1.94 & 0.61 \\
\hline & BCM2 & 1.28 & 0.097 & 0.73 & 0.00 & 0.73 & -2.28 & 0.26 \\
\hline & BCM-C & 1.18 & 0.088 & 0.64 & 0.00 & 0.64 & -1.98 & -0.09 \\
\hline & IPSL-CM4-LOOP & 1.15 & 0.077 & 0.81 & -0.34 & 0.47 & -2.01 & -0.13 \\
\hline & CNRM-CM3.3 & 0.72 & 0.057 & 0.45 & -0.32 & 0.14 & -1.25 & 0.12 \\
\hline & EGMAM+ & 0.38 & 0.025 & 0.12 & 0.19 & 0.31 & -0.56 & 0.25 \\
\hline
\end{tabular}

The sub-tables for A1B and E1 are in order of changes in T. Results are ensemble averages over all simulations in this study, except for ECHAM5-C in which data from only three of the six 20C3M and A1B simulations were used. (Results are not sensitive to this sub-sampling.) Cloud radiative forcing diagnostics were omitted from HadCM3C simulations

S. Denvil

FR 636 CNRS, UVSQ, UPMC, Institut Pierre Simon

Laplace (IPSL), 75252 Paris Cedex 05, France

J. F. Tjiputra

Department of Geophysics, University of Bergen,

Allegt. 70, 5007 Bergen, Norway 